\title{
Derin Öğrenme Mimarilerini Kullanarak Katarakt Tespiti
}

\author{
Muhammed Fatih Ağalday ${ }^{1}$, Ahmet Çınar ${ }^{2}$ \\ 1* Mardin Artuklu Üniversitesi, Meslek Yüksek Okulu, Bilgisayar Proğramcılığı, Mardin, Türkiye, (ORCID: 0000-0002-2635-0661), fatihagalday@artuklu.edu.tr \\ ${ }^{2}$ Fırat Üniversitesi, Mühendislik Fakültesi, Bilgisyar Mühendisliği Bölümü, Elazığ, Türkiye (ORCID: 0000-0003-4324-4964), acinar1972@gmail.com
}

(1st International Conference on Applied Engineering and Natural Sciences ICAENS 2021, November 1-3, 2021)

(DOI: 10.31590/ejosat.1012694)

\begin{abstract}
ATIF/REFERENCE: Ağalday, M. F. \& Çınar, A. (2021). Derin Öğrenme Mimarilerini Kullanarak Katarakt Tespiti. European Journal of Science and Technology, (28), 1428-1433.

Öz

İnsanın yaşam kalitesini olumsuz olarak etkileyen görme kayıplarını daha erken bir dönemde teşhis etmek önemlidir. İnsan yaşının ilerlemesi ile birlikte görme bozuklukları ve bazen tamamen görme kaybına neden olmaktadır. Gözün anatomik yapısında bulunan anormallikler göz hastalıklarının erken dönemlerinde göz yapısına ait görsellerle de tespit edilebilmektedir. Katarat dünyada milyonlarca insanı etkileyen görme bozukluğunun en önemli nedenidir. Otomatik tanı sistemleri ile sağlık hizmeti kullanımı hafifleyerek uzmanlara yardımcı olmayı amaçlamaktadır. Bu makalede renkli fundus görüntüler kullanılarak katarat hastalı̆ı̆na otomatik tanı sistemi ele alınmıştır. Katarat hastalığının otomatik tanımlanması için evrişimli sinir ağı (CNN) ve derin artık ağ (DRN) kullanılarak sınıflandırma yöntemi kullanılmışırı. Veri seti 5000 hastanın sağ ve sol gözlerine ait renkli fundus fotoğrafları ve doktorların her bir hastanın sağ ve sol gözüne konulmuş teşhisler için anahtar kelimler ile yapılandırılmış bir veri tabanıdır. Bu veri seti gerçek yaşamda hasta gruplarını temsil etmektedir. Çinli bir şirket olan Shanggong Medical Technology Co., Ltd. Şirketi tarafindan farklı hastane ve tıp merkezlerinden elde edilen veriler toplanmıştır. Veri setinde hastalar 8 farklı etikete sınıflandırma yapılmıştır. Renkli fundus görüntüler sayesinde farklı evrelere ait katarat semptomlarına ait özellikler bulunmaktadır. Önerilen otomatik tanı sistemi güncel sınıflandırma sistemlerine oranla daha başarılı olduğu görülmektedir. DRN yönteminin CNN yöntemine göre doğruluk oranına göre daha yüksektir. CNN modelinde doğruluk oranı \%89 civarında iken DRN modelinde doğruluk oranı \%95 olduğu görülmektedir.
\end{abstract}

Anahtar Kelimeler: Derin Öğrenme, Katarakt, Evrişimsel Sinir Ağları, Derin Kalıntı Ağı, Sınıflandırma.

\section{Cataract Detection Using Deep Learning Architectures}

\begin{abstract}
It is important to diagnose vision loss, which negatively affects the quality of life of people, at an earlier stage. With the advancement of human age, it causes visual disturbances and sometimes complete vision loss. Abnormalities in the anatomical structure of the eye can also be detected with visuals of the eye structure in the early stages of eye diseases. Cataracts are the most important cause of visual impairment affecting millions of people around the world. It aims to help experts by reducing the use of health services with automatic diagnosis systems. In this article, the automatic diagnosis system for catarrhal disease using color fundus images is discussed. Classification method using convolutional neural network (CNN) and deep residual network (DRN) was used for automatic identification of cataract disease. The dataset is a structured database with color fundus photographs of 5000 patients' right and left eyes and keywords for doctors to diagnose each patient's right and left eyes. This dataset represents real-life patient groups. Shanggong Medical Technology Co., Ltd., a Chinese company. Data from different hospitals and medical centers were collected by the company. In the data set, patients were classified into 8 different labels. Thanks to the color fundus images, there are features of cataract symptoms of different stages. It is seen that the proposed automatic diagnosis system is more successful than the current classification systems. The accuracy rate of the DRN method is higher than the CNN method. While the accuracy rate in the CNN model is around $89 \%$, the accuracy rate in the DNN model is $95 \%$.
\end{abstract}

Keywords : Deep Learning, Cataract, Convolutional Neural Networks, Deep Residual Network, Classification 


\section{Giriş}

Sağlık sistemlerinde hastalıkların daha kısa sürede teşhis edilebilmesi modern sağlık sisteminin önemli bir parçasıdır. En etkili çözümlerden biri sinir ağları kullanılarak görüntü sınıflandırılması ile teşhis yapılmasıdır. Sağlık sistemlerinin yükünü hafifletmede otomatik teşhis modelleri önemli bir rol almaktadır. Sağlık sistemlerinde hastalıkların daha kısa sürede teşhis edilebilmesi modern sağlık sisteminin önemli bir parçasıdır. En etkili çözümlerden biri sinir ağları kullanılarak görüntü sınıflandırılması ile teşhis yapılmasıdır. Sağlık sistemlerinin yükünü hafifletmede otomatik teşhis modelleri önemli bir rol almaktadır. Göz hastalıkları ve cerrahi tıp bilimi bilgisayar yardımıla tanı sistemlerinde yaygın olarak kullanılan bir alan haline geldi. Dünya sağlık örgütü tarafından tüm dünyada katarakt nedeniyle körlük oranının tüm hastalıklar arasında oranının \%51 olduğu rapor edilmiştir. Bilgisayar destekli teşhis sistemleri görme bozukluklarının teşhisinde önemli bir rol almaktadır.

Oftalmolojide, farklı cihazlardan elde edilen görüntülüler göz hastalıkları teşhisi için bilgisayar destekli tahmin etme, görünütüyü sınıflandırma için çok önemlidir. Son yıllarda yapay zekalı sistemler görüntü teşhis ve tahminlerinde önem kazanmıştır. $\mathrm{Bu}$ çalışmamızda katarakt için otomatik teşhis mimarisi ortaya koyarak fundus görüntülerinin iki sınıfta sınıflandırarak analiz yapılmıştır.

Retina görüntüleme cihazları ile elde edlen fundus görüntüler, tıbbi görüntülemenin ilk ortaya çıkışından bu yana tanı ve teşhis için geleneksel sınıflandırıcılar kullanılmıştır. Yang ve arkadaşları otomatik katarakt teşhisi için bir destek vektör makinesinden ve bir geri yayılım sinir ağından oluşturulmuş bir topluluk modeli önermektedir [1],[2] ve bu yaklaşımlar için \%90'ın üzerinde doğruluk oranına ulaşmaktadırlar. Oftalmik görüntüler için çeşitli geleneksel ve modern sınıflandırıcıları karşılaştıran kapsamlı literatür taraması bulunabilir [3],[4].

Fundus görüntülerinin derin öğrenmeye dayalı sınıflandırması için farklı yaklaşımlar bulunmaktadır [5],[6],[7]. Katarakt teşhisinde derin öğrenme yöntemleri yeterince uygulanmamıştır. Katarakt ile ilgili yapılan bir çalışmada, bulut teknolojisi ile hastaneler arasında işbirliği yapılarak yapay zeka tabanlı karar verme sistemleri oluşturulabilir [8]. Otomatik teşhis için farklı çözümler sunulmuştur. $\mathrm{Bu}$ yöntemler sayesinde katarakt hastalığının erken döneminde teşhis etmede önemli derecede başarı elde edilemektedir.

Derin öğrenmeye dayalı mimariler geliştirilerek daha yüksek doğruluk oranlarına ve düşük hesaplama maliyetine sahip algoritmalar ile teşhis ve tanıma oranı iyileştirilebilir. Katarakt ve gloucoma gibi ciddi göz hastalıklarının görsellerini temsil eden geniş bir veri setinin kullanılması göz hastalıklarının tespitinde günümüzde karşılaşılan en büyük zorluklarından biridir[9],[10]. Sonuç olarak katarakt hastalığının otomatik olarak tespit edilemesi için bu sistemlere hala ihtiyaç duyulmaktadır.

\subsection{Veri Seti}

$\mathrm{Bu}$ çalışmada kullanılan veritabanı Shanggong Medical Technology Co. şirketi tarafindan Çin'deki farklı hastanelerden/sağlık merkezlerinden elde edilen gerçek hasta bilgilerini temsil eden 5000 hastanın sağ ve sol gözüne ait 6392 renkli fundus görüntülerinden oluşmaktadır. Her bir fundus görseli farklı çözünürlük ve piksel değerlerine sahiptir. Uzman doktorların her bir renki fundus görüntülerini tanımlayan teşhis anahatar kelimler ile yapılandırılmış olup Kaggle Ocular Disease Recognition veritabanı elde edilmiştir. Hastalara ait fundus görüntüler Normal (N), Diyabet (D), Glokom (G), Katarakt (C), Yaşa Bağlı Makula Dejenerasyonu (A), Hipertansiyon (H), Patolojik Miyop (M), Diğer hastalıklar /anormallikler (O) olmak üzere sekiz etikette sınıflandırılmıştır. Yapılan çalışmada sınıflandırma için kullanılan her bir sınıfa ait örnek fundus görüntüler Şekil 1' de verilmiştir. Veri kümesi, farklı aşamadaki katarakt hastaları için çeşitli kümeler içerir ve bu yapay zeka tabanlı bir sınıflandırma sisteminin başarısı için çok önemlidir.

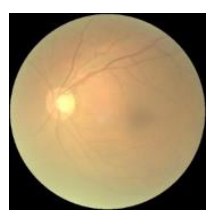

(a) Katarakt

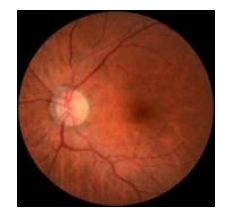

(b) Katarakt Olmayan
Şekil 1. Her Sınıf için Örnek Görseller

\section{Yöntem}

\subsection{Evrişimsel Sinir Ağı (CNN)}

$\mathrm{Bu}$ çalışmada, ikili sınıflandırma problemini çözmek için Evrişimli Sinir ağı kullanılmıştır. Evrişimsel Sinir Ağları [11], evrişim katmanları olarak adlandırılan ilk katmanların hiyerarşik bir özellik çıkarıcı olarak hareket edecek şekilde yapılandırıldığ çok katmanlı algılayıcılarından oluşan bir yapıya sahiptir. Yapay sinir ağının kullanılan yapısı şekil 2'de görülebilir. Konvolüsyonel sinir ağı, öznitelik çıkarma ve sınıflandırma olmak üzere iki ana bölüm olarak yapılandırılmıştır. Özellik çıkarma bölümünde her bir blokta 1 konvolüsyon ve 1 havuzlama katmanında oluştan toplamda 5 blok bulunmaktadır. Sinıflandırma kısmında ise tam bağlantılı katman bulunmaktadır. CNN'nin detayları kalan bölümlerde açıklanacaktır.
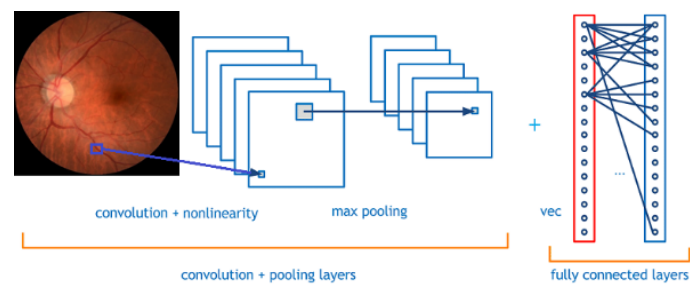

Şekil 2. Evrişimsel Sinir Ă̆ının Yapısı

Uzman doktorların her bir hastanın sağ ve sol gözüne ait teşhis için kullandıkları anahtar kelimeler ile veri kümesindeki renki fundus görüntüleri eşleştirmek için anahtar kelimelerin olduğu csv dosyası kullanılır. Her bir hastanın sağ ve sol göze ait görüntülerden hangilerinin katarat olduğu farklı değişkenlerde tutulurarak daha sonra tüm katarat anahtar bilgisine sahip sağ ve sol göz bilgisi tek bir değişkende tutulur. Aynı işlemler hastaların sağlıklı olan sağ ve sol gözlerine ait görüntüler içinde uygulanır. Renkli fundus veri kümesindeki tüm görüntüler 0-255 arasında ölçeklendirilir ve görüntü boyutu 224 x 224 formatına dönüştürülür. Hem Evrişimli Sinir Ağı hem de Derin Kalıntı Ağı için aynı veri kümesindeki veriler kullanılır.

Evrişimli Sinir Ağı yönteminde öznitelik çıkarımı, geleneksel öznitelik çıkarma yöntemleri yerine evrişim çekirdekleri 
kullanılarak yapılır. Çeşitli Evrişimsel katmanlardaki çoklu filtreler, bir sonraki aşamada CNN'nin daha derin katmanlarındaki tamamen bağlı ağlara beslenecek olan ayırt edici özellikleri çıkarır. Özellik çıkarımı yapabilmek için şekil 2'de görüldüğü gibi farklı sayılarda blok kullanılmaktadır. Tüm blokta evrişim ve havuz katmanları bulunmaktadır. Evrişim katmanlarındaki çekirdek sayısı ve boyutları farklı olabillir. Havuzlama katmanındaki çekirdek boyutu 2 × 2 veya 3 X 3 gibi ve daha farklı şekillerde havuzlama katmanları da kullanılmaktadır. Blok yapılarda farklı sayılarda katmanlar olabilir. Örneğin her blokta farklı sayılarda çekirdek yapısı ve ayrıca her blokta tanH, softmax ve ReLU gibi farklı aktivasyon fonksiyonları kullanılabilir. Her bir filtrelemede öznitelik haritaları olarak da adlandırılan öznitelikler çıkarılır. Bu çalışmada kullanılan klasik CNN yapısı evrişim katmanı ilk blokta 64 , ikinci blokta 128 , üçüncü blokta 256 ve diğer blokta 512 çekirdekli blok yapısı bulunmaktadır. Havuzlama katmanında çekirdek boyutu 2 × 2 ve her katmanda ReLU aktivasyon fonksiyonu kullanılmıştır. Klasik CNN algoritması sınıflandırma işlemi yapılırken evrişimsel sinir ağının son bloğu, görüntünün katarat olup olmadığını sınıflandırmak için kullanılan çıkış nöronlarını içerir. En sondaki bloğa tam bağlı blok denir. Tam bağlantılı blokta iki katmadan oluşmaktadır. $\mathrm{Bu}$ katman ReLU aktivasyonlu ve belirlenen nörona sahiptir ve son katman lineer aktivasyonlu 2 nörona sahiptir. Her örnek katarat ya da katarat olmayan olarak sınıflandırılır. CNN, ikili çapraz entropi kaybı fonksiyonu ile eğitilmiştir ve adaptive momentum (adam) optimizasyonu ile optimize edilmiştir. Adam optimizasyonunda sabit öğrenme oranı yoktur.

\subsection{Derin Artık Ăg (DRN)}

Derin Artık Ağ (DRN) ve geleneksel Evrişimli Sinir Ağları $(\mathrm{CNN})$ arasındaki en önemli fark, DRN'lerin eğitim sırasında gradyanların erken katmanlara geri yayılması için açı bir yol sağlamasıdır [12]. CNN'de, H(x), şekil 5'teki CNN'nin bir kısmını haritalamaktadır. DRN'de, $\mathrm{H}(\mathrm{x}), \mathrm{F}(\mathrm{x})+\mathrm{x}$ olarak tanımlanır, burada $\mathrm{x}$, ağın bir bölümünün girişidir. $\mathrm{Bu}$ nedenle önceki katmanlardaki değerler daha kolay tanımlanabilir.

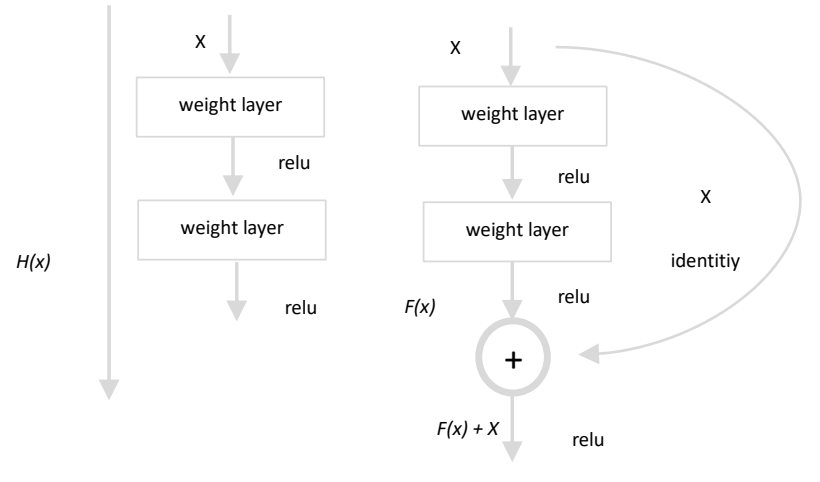

Şekil 3. Sol: Geleneksel CNN, Sağ: Artık Blok

Derin Artık Ağ, şekil 4'teki temel artık bloklardan oluşur. Temel artık blok mimarisi, sırasıyla evrişim, toplu normalleştirme (BN), ReLU, dropout, Evrişim, toplu normalleştirme katmanlarından oluşur. ReLU, kimlik fonksiyonu (X) ile eklendikten sonra bloğun sonuna uygulanır.

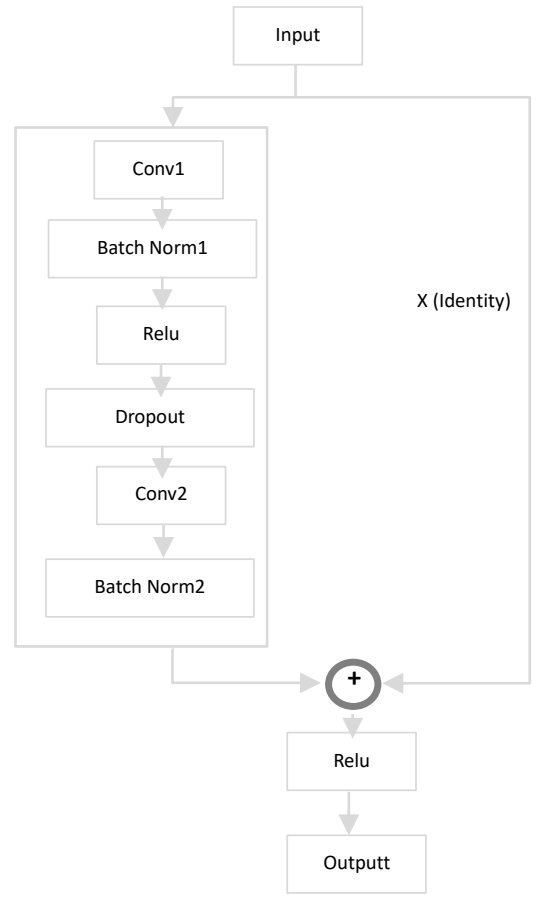

Şekil 4. Temel Artık Blok

Deep Residual Network mimarisi, Şekil 5'de görüldüğü gibi sırasıyla evrişim, Batch normalleşme, ReLU, Havuzlama, Block$1,2,3, \ldots$ havuzlama ve tam bağlantılı katman serilerinden oluşmaktadır.

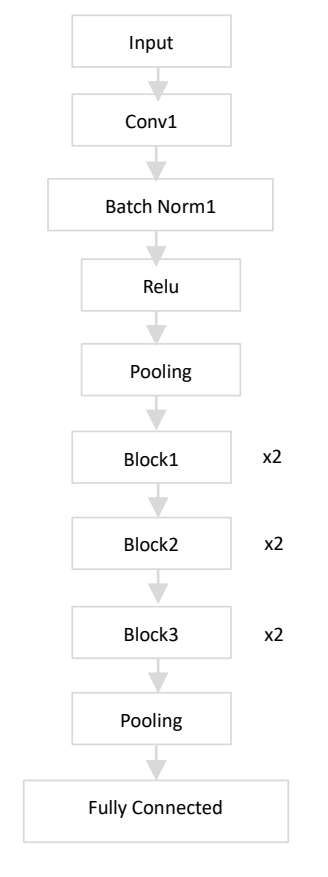

Şekil 5. Derin Artık A

Önerilen algoritmada DRN öznitelik çıkarma kısmı CNN gibidir. Bu, geleneksel bir özellik çıkarma yöntemi olmadığı anlamına gelir. DRN'de özellik çıkarımı için evrişimli katmanlar kullanılır. DRN yapısı için özellik çıkarımına örnek olarak şekil 5 'te görüldüğü gibi üç tip blok kullanılmıştır. Her bir evrişim katmanı farklı çekirdek sayısı ve çekirdek boyutuna sahip olabilir. Tam tersi bir durum da söz konusu her blok aynı evrişim katmanı içeren mimariye de sahip olabilir. Blokların mimarisi aynı olsa bile bloklardaki çekirdek sayıları birbirinden farklı 
olabilir. Örnek olarak Blok1 yapısında ilk evrişim katmanında 64 çekirdek sayısına sahip iken ve ikinci evrişim katmanının çekirdek sayısı 128 olabilir. Bloklardaki tüm evrişimli katmanlarda aynı şekilde farklı çekirdek boyutları kullanılabilir. Klasik CNN yapsında olduğu gibi tüm katmanlar için Relu gibi farklı aktivasyonlar tercih edilebilir. Özellik çıkarımından sonra havuzlama uygulanır ve en son DRN'nin sınıflandırma kısmı olacaktır. Evrişimsel sinir ağının son bloğu, görüntülerde katarakt olup olmadığını sınıflandırmak için kullanılan çıkış nöronlarını içerir. Son bloğa tam bağlı (FC) blok denir. DRN, çapraz entropi kaybı fonksiyonu ile eğitilebilir ve rmsprop, adam,SGV gibi optimizer ile optimize edilebilir.

\section{Kullanılan Yöntem}

Katarakt hastalığını etkili bir şekilde tanımlamak için derin bir sinir ağı tabanlı sınıflandırma sistemi önerilmiştir. Önerilen metodoloji, Şekil 6'da gösterildiği gibi adımlar takip edilerek katarakt hastalığının otomatik olarak sınıflandırılması amaçlamaktadır. Veri seti Kaggle veri tabanından elde edilmiştir ve 7000 renkli fundus görüntüsünden oluşmaktadır (594 katarakt ve geri kalan görüntüler katarakt olmayan). Diyagramın ilk adımı, veri setinden oluşan tüm görüntülerin girişini gösterir. Renkli fundus görüntüleri daha sonra güçlü olan derin özellik çıkarma mimarisi olan VGGNet ile derin öğrenme tekniğinin ön eğitimi için kullanıldı. Katarakt görüntülerinin tespiti için VGGNET derin öğrenme mimarisinden elde edilen özellikler kullanılmıştır. Tespit aşamasında, tüm verilerin \%80'i eğitim ve doğrulama için ve ayrıca tüm verilerin \%20'si test için rastgele kullanılmıştır.

Önerilen yaklaşımın yardımıyla, çok etkili ve düşük maliyetli bir katarakt sistemi teşhisi tasarlanmıştır. Ayrıca katarakt tanısının farklı evrelerde konulması için dikkate değer bir doğruluk oranı sağlanmakta ve böylece vakada yanlış tanıdan kaçınılmaktadır. Yukarıda belirtilen adımlar, veri kümesini kullanarak kataraktı doğru bir şekilde tanımlamak için önerilen metodoloji tarafından yürütülmüştür.

$\mathrm{Bu}$ çalışma python ortamında gerçekleştirilmiştir. Önceden eğitilmiş VGGNet yapısının eğitimi, transfer öğrenme kullanılarak gerçekleştirildi. Eğitim sürecinde batch boyutu 32 ve epoch sayıs1 100'dür. Her epoch için 30 olmak üzere toplam 3000 iterasyon gerçekleştirilmiştir. Başlangıç için öğrenme oranı 0.001 'dir ve optimize edici olarak Adam seçilmiştir. Bu çalışmada validasyon veri seti, eğitim veri setinin \%20'si olarak belirlenmiştir. Bu çalışmada eğitilmiş CNN mimarilerinin eğitim, doğrulama ve test çizelgeleri Şekil 6'da verilmiştir.

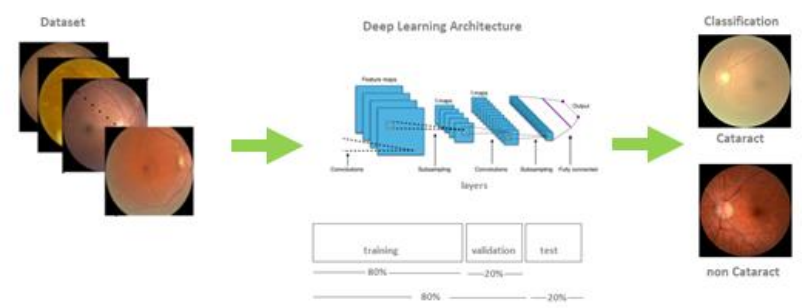

Şekil 6. Önerilen Metodolojinin Blok Şeması

Derin Öğrenme Mimarilerinde Transfer öğrenimi, daha önce eğitilmiş mimarilerin son katmanı değiştirerek yeniden eğitilmesine olanak tanır. Bu çalışmada, bir önceki katmanın parametrelerini tutan ve bu mimarilerin son katmanını kaldıran, ardından son katmanı yeniden eğiten transfer öğrenme yöntemi kullanılmıştır. Son katmandaki çıktı düğümlerinin sayısı, sınıflandırılan veri kümesi kategorilerinin sayısına eşittir.

$\mathrm{Bu}$ mimaride ReLU etkinleştirme işlevini kullanan evrişim katmanı içerir. Maksimum havuzlama işlemi evrişimden sonra gerçekleştirilir. Softmax katmanı, birkaç tam bağlantılı sinir ağ katmanıyla bu mimaride sınıflandırma yapmak için kullanılır. VGGNet sağlam bir mimariye sahip olsa da AlexNet ile benzer bir yapıya sahiptir. VGGNet'in genel yapısına bakıldığında son zamanlarda yapılan çalışmalarda kullanılan mimarin yerini aldığ görülmektedir [13],[14]. VGGNet'in mimarisinde sadece $3 \times 3$ boyutlu filtreler kullanılmaktadır. VGGNet yapısında kullanılan filtreler genellikle bir görüntünün özniteliğini çıkarmak için tercih edilmektedir. VGGNet modelinin son katmanı dışındaki tüm katmanlar dondurulmuş ve katarakt hastalığı tespiti için ağırlık değerleri aktarılmıştır. Ayrıca, VGGNet beş VGG bloğuna sahiptir ve bu blokların her biri iki Convolution ve Relu katmanı içerir. Önerilen metodolojinin genel performansını hesaplamak için istatistiksel metrikler doğruluk, duyarlılık, kesinlik ve Hata oranları olarak sunulmaktadır. Her metrik için matematiksel denklem sırasıyla aşağıda verilmiştir.

$$
\begin{gathered}
\text { Doğruluk }=\frac{\mathrm{TP}+\mathrm{TN}}{\mathrm{TP}+\mathrm{FP}+\mathrm{FN}+\mathrm{TN}} \\
\text { Hassasiyet }=\frac{\mathrm{TP}}{\mathrm{TP}+\mathrm{FN}} \\
\text { Kesinlik }=\frac{\mathrm{TP}}{\mathrm{TP}+\mathrm{FP}} \\
\text { Hata }=\frac{\mathrm{FP}+\mathrm{FN}}{\mathrm{TP}+\mathrm{FP}+\mathrm{FN}+\mathrm{TN}}
\end{gathered}
$$

Yukarıda verilen denklemlerde TP, FP, TN ve FN bir karışıklık matrisinin sırasıyla Doğru Pozitif, Yanlış Pozitif, Doğru Negatif ve Yanlış Negatif'i ifade etmektedir. Önerilen sistemin uygulaması için Intel (R) Core (TM) i7-10750H CPU $2.60 \mathrm{GHz}$, 24 GB 2666MHz RAM ve 6GB GDDR6 NVIDIA GeForce GTX 1660 Ti grafik işlem birimi donanımı üzerinde yürütülmektedir.

\section{Tartışma}

$\mathrm{Bu}$ çalışmada klasik CNN ile derin öğrenme mimarisi kullanılarak katarakt hastalığının teşhisi gerçekleştirilmiştir. Önerilen bu metodoloji, geniş bir fundus renkli görüntü veri seti üzerinde uygulanmaktadır ve sistemlerin performansı, alt bölümde evrensel metrikler ile kıyaslama açısından sunulmaktadır. Sonuç olarak, klasik CNN ile önceden eğitilmiş mimariyi kullanan ve önerilen sistemin VGGNet doğruluk oranları Tablo 1'de verilmiştir. Tablo 1'deki doğruluk sonuçları göz önüne alındığında, önerilen her iki metodoloji de literatürde tanitılan tanımlama sistemlerine kıyasla dikkate değer sonuçlar olan \%90'ın üzerinde doğruluk oranlarına ulaşmaktadır [15], [16], [17].

Tablo 1. Istatistiksel performans ölçütleri

\begin{tabular}{|l|c|c|}
\hline $\begin{array}{l}\text { Performans } \\
\text { Metrikleri }\end{array}$ & $\begin{array}{l}\text { VGGNet } \\
\text { Mimarisi }\end{array}$ & $\begin{array}{l}\text { Klasik CNN } \\
\text { Mimarisi }\end{array}$ \\
\hline Doğruluk (\%) & 94.3 & 90 \\
\hline Duyarlılık & 0.94 & 0.89 \\
\hline Kesinlik & 0.95 & 0.90 \\
\hline Hata & 0.05 & 0.1 \\
\hline
\end{tabular}

VGGNet karmaşıklık matrisinde katarat olmayan doğru tespit sayısı 565, yanlış tespit sayısı 36 , katarat olanların doğru tespit 
sayısı 561, yanlış tespit sayısı 32 olarak elde edilirken, klasik CNN ile katarat olmayan doğru tespit sayısı 543, yanlış tespit sayısı 62, katarat olanların doğru tespit sayısı 532, yanlış tespit sayıs 57 olarak elde edilmiştir. Değerlendirme VGGNet mimarisi için karışıklık matrisinde, katarakt testi görüntülerinin \%94,6'sı gerçek pozitif (TP) ve \%5,4'ü yanlış pozitif (FP) olarak tespit edilirken, katarakt olmayan görüntülerin \%94'ü doğru negatif (TN) ve \%6'sı yanlış negatif (FN) olarak hesaplanır. Benzer şekilde CNN mimarisi için karışıklık matrisi dikkate alındığında, katarakt olmayan görüntülerin \% 89,76's1 (TP) ve \%10,24'ü (FP) olarak bulunurken, katarakt test görüntülerinin \%90,32'si (TN) ve \%9,68'i (FN) olarak hesaplanmıştır. Bu analizler, halk sağlığı sistemleri ağlarına entegrasyonu durumunda önerilen sistemlerin performansı için kapsamlı bir perspektif sağlar.

Önerilen metodolojinin performans metrikleri değerlendirilmiş ve Tablo 1' de sunulmuştur, duyarlılığın iki mimari için eşit olduğu, VGGNet mimarisi için kesinliğin 0.95 ve Klasik CNN mimarisi için 0.90 olduğu görülmektedir. Duyarlılık metriği her iki mimari için eşit olarak elde edilir. İki mimari için kesinlik değerleri değerlendirildiğinde, retina görüntülerinden kataraktı tespit etmede VGGNet mimarisinin Klasik CNN mimarisine göre daha başarılı olduğu görülmektedir.

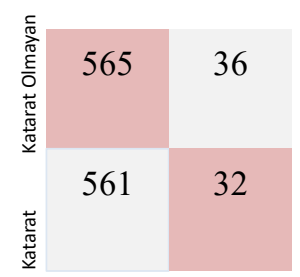

(a) VGGNet Mimarisi

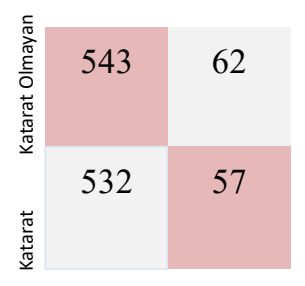

(b) Klasik CNN Mimarisi

\section{Şekil 7. Kullanılan Mimarilerm için Karışıklık Matrisi}

\section{Sonuçlar}

Son yıllarda insan gözü hastalığının otomatik tespiti ve yönetimi için yapay zeka tabanlı çeşitli çalışmalar yapılmaktadır. $\mathrm{Bu}$ çalışmalar, kayda değer doğruluk oranlarına ulaşan otomatik tanımlama ve yönetim sistemlerini devreye sokarak halk sağlı̆̆ kuruluşlarının ve hastanelerin yükünü azaltarak önemli bir boşluğu doldurmuştur. Son çalışmalar, derin öğrenme tabanlı mimarilerin, geleneksel sınıflandırıcılara kıyasla oftalmolojik hastalıkların teşhisi için daha kabul edilebilir performans gösterdiğini göstermektedir. Katarakt tespit sistemlerinin yaygınlaşması ve bu sayede körlüğün önlenmesi için bilgisayar destekli teşhis sistemlerinin teletıp sistemlerine entegre edilmesi önemli bir noktadır. $\mathrm{Bu}$ bağlamda, bilgisayar destekli karar/yönetim sistemlerinin sağlık sistemlerine gerçek zamanlı entegrasyonu için önceki bölümlerde tartışılan çeşitli uygulama parametrelerinin dikkate alınması gerekmektedir. Bu çalışmada, katarakt hastalıklarının otomatik teşhisi için VGGNet gibi sağlam, önceden eğitilmiş derin öğrenme mimarisi kullanılmıştır. Farklı evrelerdeki katarakt hastalarının çeşitli renkli fundus görüntülerini içeren veri seti, yeni, benzersiz ve geniş bir veri setidir. $\mathrm{Bu}$ nedenle, bu özel veri kümesi üzerinde yürütülen mimarilerin değerlendirilmesi, hastalığın otomatik teşhisinin olasılıkları ve performans parametreleri için açık bir perspektif verir. Çalışma, önerilen metodolojiyi katarakt hastalarından elde edilen geniş bir fundus görüntü veri seti üzerinde uygulayarak literatüre özgün bir katkı sağlamıştır. Metodoloji, katarakt için otomatik teşhis görevini \%95'in üzerinde umut verici doğruluk oranlarıyla yerine getirdi. Bu sonuç, katarakt hastalığı için sağlam sınıflandırma sistemleri açısından oldukça iyi sonuçlar ortaya koymaktadır.

\section{Kaynakça}

[1] Yang J J, Li J, Shen, R, Zeng Y, He J et al.(2016). Exploiting ensemble learning for automatic cataract detection and grading. Computer Methods and Programs in Biomedicine; 124: 45-57. doi:10.1016/j.cmpb.2015.10.007

[2] Yang M, Yang J J, Zhang Q, Niu Y, Li J. Classification of retinal image for automatic cataract detection, In: IEEE International Conference on e-Health Networking, Applications Services; Lisbon, Portugal; 2013. pp. 674-679. doi:10.1109/HealthCom.2013.6720761

[3] Wang Liming, Zhang K, Liu X, Long E, Jiang J, An Y et al. (2017). Comparative analysis of image classification methods 5 for automatic diagnosis of ophthalmic images. Scientific Reports; 7: 1-11. doi:10.1038/srep41545

[4] Gali H E, Sella R, Afshari N A. ( 2019). Cataract grading systems: a review of past and present. Current opinion in ophthalmology; $\quad 30(1)$ : 13-18. doi: 10.1097/ICU.0000000000000542

[5] Grewal P S, Oloumi F, Rubin U, Tennant M T S. (2018). Deep learning in ophthalmology: a review. In Canadian Journal of Ophthalmology; 53(4): 309313.doi:10.1016/j.jcjo.2018.04.019

[6] He J, Li C, Ye J, Qiao Y, Gu L. (2021). Multi-label ocular disease classification with a dense correlation deep neural network. Biomedical Signal Processing and Control ; 63: doi:10.1016/j.bspc.2020.102167

[7] Yoo T K, Ryu I H, Kim J K, Lee I S, Kim J S et al. (2020). Deep learning can generate traditional retinal fundus photographs using ultra-widefield images via generative adversarial networks. Computer Methods and Programs in Biomedicine; 197: doi:10.1016/j.cmpb.2020.105761

[8] Long E, Lin H, Liu Z, Wu X, Wang L, et al. (2017). An artificial intelligence platform for the multihospital collaborative management of congenital cataracts. Nature biomedical engineering ; 1(2): 1-8. doi: doi.org/10.1038/s41551-016-0024

[9] Zhang H, He Z. (2019). Automatic cataract grading methods based on deep learning. Computer Methods and Programs in Biomedicine; 182: doi:10.1016/j.cmpb.2019.07.006

[10] Yanagihara R T, Lee C S, Ting D S W, Lee A Y. (2020). Methodological challenges of deep learning in optical coherence tomography for retinal diseases: a review. Translational Vision Science \& Technology; 9(2): 11-11. doi: 10.1167/tvst.9.2.11

[11] Y. Lecun, L. Bottou, Y. Bengio, and P. Haffner, (Nov. 1998). "Gradient-based learning applied to document recognition," Proc. IEEE, vol. 86, no. 11, pp. 2278-2324,

[12] He, K., Zhang, X., Ren, S., Sun, J. (2016). Deep Residual Learning for Image Recognition. IEEE Conference on Computer Vision and Pattern Recognition (CVPR) , 770778.

[13] Liefers B, Venhuizen F G, Schreur V, van Ginneken B, Hoyng $\mathrm{C}$ et al. (2017). Automatic detection of the foveal 
center in optical coherence tomography. Biomedical Optics Express; 8(11): 5160. doi:10.1364/boe.8.005160

[14] Xu X, Zhang L, Li J, Guan Y, Zhang L. (2020). A Hybrid Global-Local Representation CNN Model for Automatic Cataract Grading. IEEE Journal of Biomedical and Health Informatics; 24(2): 556-567. doi:10.1109/JBHI.2019.2914690

[15] Gour N, Khanna P. (2020). Multi-class multi-label ophthalmological disease detection using transfer learning based convolutional neural network. Biomedical Signal Processing and Control ; doi: doi.org/10.1016/j.bspc.2020.102329

[16] Pratap T, Kokil P. (2019). Computer-aided diagnosis of cataract using deep transfer learning. Biomedical Signal Processing and Control; 53: 1-8. doi: 10.1016/j.bspc.2019.04.010

[17] Sengupta S, Singh A, Leopold H A, Gulati T, Lakshminarayanan, V. (2020). Ophthalmic diagnosis using deep learning with fundus images - A critical review. Artificial intelligence in medicine ; 102: doi: 10.1016/j.artmed.2019.101758 